\title{
A simple approach to describe hadron production rates in $\mathrm{e}^{+} \mathrm{e}^{-}$annihilation *
}

\author{
Yi-Jin Pei \\ ${ }^{1}$ CERN, CH-1211 Geneva 23, Switzerland (e-mail: peiyj@vxcern.cern.ch) \\ ${ }^{2}$ I. Physikalisches Institut der RWTH, D-52074 Aachen, Germany
}

\begin{abstract}
We show that, based on the idea of string fragmentation, the production rates of light flavored mesons and baryons originating from fragmentation can be described by the spin, the binding energy of the particle, and a strangeness suppression factor. Apart from a normalization factor, $\mathrm{e}^{+} \mathrm{e}^{-}$data at different center-of-mass energies can be described simultaneously. Applying to the heavy flavor production, we find that our predictions are in good agreement with data.
\end{abstract}

\section{Introduction}

The soft processes of hadronization are not calculable with a perturbative approach and instead rely currently on phenomenological models. The most successful of these models are the string 1 and the cluster $\mathrm{a}$ fragmentation models, implemented in the Monte Carlo programs JETSET ${ }^{3}$ and HERWIG 4 , respectively. However, these models either require a large number of free parameters in order to reproduce the measured hadron production rates (JETSET), or do not give a satisfactory description of baryon data (HERWIG). A review may be found in Ref.

In this paper, we first show some regularities in the hadron production rates measured at LEP. Then based on the idea of string fragmentation, we deduce a simple formula to describe the LEP data. The formula is also applied to data obtained at center-of-mass energies around $10 \mathrm{GeV}$ and 29$35 \mathrm{GeV}$, and to the heavy flavor production.

\section{Overview of LEP data}

Thanks to excellent performance of the detectors and high statistics available, very careful work by all four LEP experiments has given a very complete picture of the production of identified particles from $\mathrm{e}^{+} \mathrm{e}^{-}$annihilation. The measured production rates per hadronic $\mathrm{Z}$ event for the identified particles at LEP 1 are listed in Table 1 .

Studies of general features of particle production, such as the fraction $V /(V+P)$ of mesons produced in spin- 1 states and the strangeness suppression factor $\gamma_{s}=\mathrm{s} / \mathrm{u}$, provide useful information about the fragmentation process. The ratio $V /(V+P)$ for primary mesons is expected to be

\footnotetext{
* Presented at ICHEP'96, July, 1996, Warsaw, Poland
}

equal to 0.75 from simple spin counting. From the rates and the primary fractions given in $\mathrm{Ta}-$ ble 11 and Ref. 6 , we obtain a value of $0.46 \pm 0.04$, $0.42 \pm 0.03,0.56 \pm 0.04$ and $0.75 \pm 0.04$ for $u(d)$-, $\mathrm{s}-, \mathrm{c}-$ and b-mesons respectively. The low value of $V /(V+P)$ for light flavored mesons could be explained by mass differences between the vector and pseudoscalar mesons, i.e. by the relatively larger binding energy of pseudoscalar mesons. For c-mesons the measured ratio of $V /(V+P)$ is also low, while for b-mesons it agrees well with the expected value of 0.75 . We will discuss this later in more detail.

Figure 1 shows the production rates of primary hadrons (measured rate $\times$ fraction from JETSET as listed in Table 1), divided by the spin factor $(2 \mathrm{~J}+1)$, as a function of the hadron mass. We see similar behavior f (steps) for hadrons belonging to the same multiplet, such as $\left(\rho / \omega, \mathrm{K}^{*}, \phi\right),(\mathrm{p}, \Lambda, \Sigma, \Xi),\left(\Delta, \Sigma^{*}, \Xi^{*}, \Omega\right)$ and $\left(\mathrm{f}_{2}, \mathrm{~K}_{2}^{*}, \mathrm{f}_{2}^{\prime}\right)$. The mass difference of hadron pairs which are in the same multiplet but differ by one in the strangeness is in the range of $0.1-0.15 \mathrm{GeV}$ (except $m_{\mathrm{K}}-m_{\pi}$ ). This is close to the mass difference between the $\mathrm{s}$ and the $\mathrm{u}(\mathrm{d})$ quark, showing that the binding energy of hadrons in each pair is about the same. Unlike the case of $V /(V+P)$, one could expect the $\gamma_{s}$ value determined from different hadron pairs is about the same.

In the string fragmentation model, one expects the strangeness suppression factor $\gamma_{s}$ to be around 0.3. This parameter can be measured from the production rates of strange compared with non-strange hadrons, and from the momen-

${ }^{a}$ From Fig. 1 one can see that it is not possible to describe all the data with just an exponential function of the hadron mass (or mass squared). 
Table 1: Average particle production rates (excluding charge conjugates and antiparticles), compared to the calculated values (see next section). The fraction of primary hadrons obtained from the fit and JETSET is also shown.

\begin{tabular}{|lllll|}
\hline Particle & $\begin{array}{l}\text { Rate } \\
\text { Measured }\end{array}$ & $\begin{array}{l}\text { Rate } \\
\text { Calc. }\end{array}$ & $\begin{array}{l}\text { Prim. } \\
\text { Frac. } \\
\text { Calc. }\end{array}$ & $\begin{array}{l}\text { Prim. } \\
\text { Frac. } \\
\text { JETSET }\end{array}$ \\
\hline$\pi^{0}$ & $9.19 \pm 0.73$ & 9.77 & 0.16 & 0.14 \\
$\pi^{+}$ & $8.53 \pm 0.22$ & 8.70 & 0.18 & 0.16 \\
$\mathrm{~K}^{0}$ & $1.006 \pm 0.017$ & 1.008 & 0.25 & 0.30 \\
$\mathrm{~K}^{+}$ & $1.185 \pm 0.065$ & 1.100 & 0.23 & 0.27 \\
$\eta$ & $0.95 \pm 0.11$ & 0.85 & 0.30 & 0.33 \\
$\eta^{\prime}$ & $0.22 \pm 0.07$ & 0.11 & 0.59 & 0.79 \\
\hline $\mathrm{f}_{0}(980)$ & $0.140 \pm 0.034$ & 0.080 & 0.99 & 0.93 \\
\hline$\rho^{0}$ & $1.29 \pm 0.13$ & 1.12 & 0.47 & 0.54 \\
$\mathrm{~K}^{* 0}$ & $0.380 \pm 0.021$ & 0.390 & 0.49 & 0.60 \\
$\mathrm{~K}^{*+}$ & $0.358 \pm 0.034$ & 0.395 & 0.50 & 0.60 \\
$\omega$ & $1.11 \pm 0.14$ & 1.05 & 0.48 & 0.57 \\
$\phi$ & $0.107 \pm 0.009$ & 0.092 & 0.64 & 0.70 \\
\hline $\mathrm{f}_{2}(1270)$ & $0.25 \pm 0.08$ & 0.19 & 0.78 & 0.96 \\
$\mathrm{~K}_{2}^{*}(1430)^{0}$ & $0.095 \pm 0.035$ & 0.051 & 1.00 & 0.98 \\
$\mathrm{f}_{2}^{\prime}(1525)$ & $0.020 \pm 0.008$ & 0.018 & 1.00 & 0.98 \\
\hline $\mathrm{p}$ & $0.49 \pm 0.05$ & 0.54 & 0.12 & 0.56 \\
$\Lambda$ & $0.186 \pm 0.008$ & 0.165 & 0.12 & 0.44 \\
$\Sigma^{0}$ & $0.0355 \pm 0.0065$ & 0.0387 & 0.40 & 0.86 \\
$\Sigma^{+}$ & $0.044 \pm 0.006$ & 0.037 & 0.42 & 0.88 \\
$\Xi^{-}$ & $0.0129 \pm 0.0007$ & 0.0118 & 0.41 & 0.75 \\
\hline$\Delta^{++}$ & $0.064 \pm 0.033$ & 0.069 & 0.69 & 0.95 \\
$\Sigma(1385)^{+}$ & $0.011 \pm 0.002$ & 0.016 & 0.91 & 0.92 \\
$\Xi(1530)^{0}$ & $0.0031 \pm 0.0006$ & 0.0050 & 0.94 & 0.94 \\
$\Omega^{-}$ & $0.0008 \pm 0.0003$ & 0.0015 & 0.88 & 0.92 \\
\hline$\Lambda(1520)$ & $0.0107 \pm 0.0014$ & 0.0129 & 0.71 & - \\
\hline
\end{tabular}

tum spectrum of strange mesons. The results are very consistent with the expectation (a review may be found in Ref.9). This suggests that the strangeness suppression occurs at the quark level. In the following we consider that the particle production proceeds in two stages, namely quark pair production in the color string field and successive recombination.

\section{Analysis}

Quark pair production in the color string field can be considered as a tunneling process. The probability to produce a $q \bar{q}$ pair is proportional to $\exp \left(-\pi m_{q}^{2} / \kappa\right)$, where $m_{q}$ is the (constituent) quark mass, and $\kappa$ the string constant. We assume that the probability of quarks recombined to a hadron with the mass $M_{h}$ is proportional to $\exp \left(-E_{\text {bind }} / T\right)$, where $E_{\text {bind }}=M_{h}-\sum_{i} m_{q_{i}}$ is the hadron binding energy $\uparrow$, and $T$ the effective temperature in hadronization. The production rates of light flavored mesons and baryons from fragmentation can be described as

$$
<N>=C \cdot \frac{2 J+1}{C_{B}} \cdot\left(\gamma_{s}\right)^{N_{s}} \cdot \mathrm{e}^{-\frac{E_{b i n d}}{T}},
$$

\footnotetext{
${ }^{b}$ It can be ascribed to the hyperfine interaction 10
}

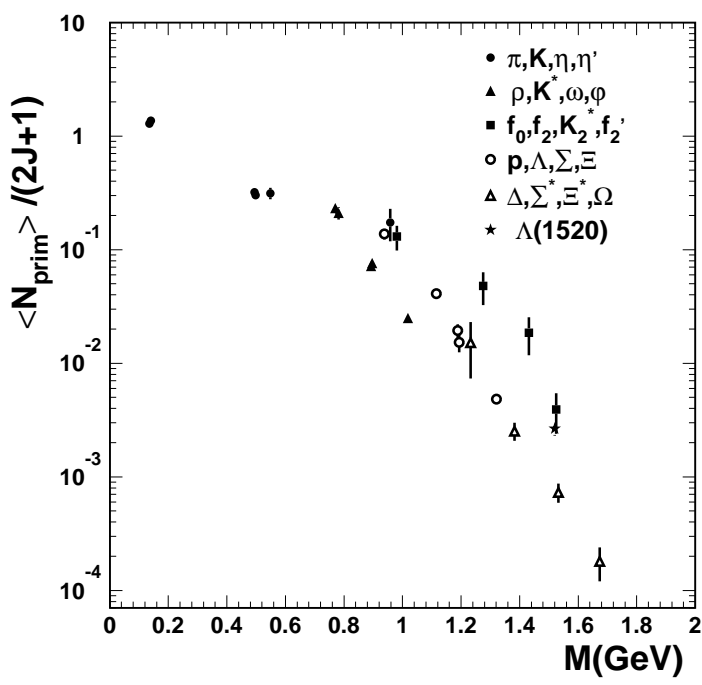

Figure 1: Production rates of primary light flavored hadrons at LEP energy, divided by the spin factor $(2 \mathrm{~J}+1)$, as a function of the hadron mass.

where $\gamma_{s}=\exp \left(-\pi\left(m_{s}^{2}-m_{u}^{2}\right) / \kappa\right)$ is the strangeness suppression factor, $N_{s}$ the number of strange quarks contained in the hadron, and $J$ the spin of the hadron. $C$ is an overall normalization factor, and $C_{B}$ is the relative normalization factor between mesons and baryons (for mesons $C_{B}=1$ ). Equation 11 can also be applied to mixed states of the $\mathrm{SU}(3)$ octet and singlet, such as $\eta$ and $\eta^{\prime}$, by adding up the $u \bar{u}(d \bar{d})$ and $s \bar{s}$ contributions. For this purpose we yse the mixing formulae and angles given in Ref. 11 .

Total hadron rates are calculated as follows. At first the number of light flavored hadrons produced from fragmentation is calculated by using Eq.11. For hadrons which contain a primary quark $q$, we use Eq.1 to determine their relative ratios, and then get their rates by normalizing the sum of the rates to the $q \bar{q}$ fraction, $\Gamma_{q \bar{q}} / \Gamma_{h a d}$, which can be calculated by the Standard Model. All light flavored hadrons up to a mass of $2.5 \mathrm{GeV}$ in the meson and baryon summary table of Ref. 11 are included in the calculation. In the next step we let all these primary hadrons decay according to their decay channels and branching ratios given in Ref.11. The decay chain stops when $\mu, \pi, \mathrm{K}^{ \pm}, \mathrm{K}_{L}^{0}$ or stable particles are reached.

In the fit we choose $\gamma_{s}, \Delta m=m_{s}-m_{u}, T, C$ and $C_{B}$ as free parameters (we assume $m_{u}=m_{d}$ ).

\footnotetext{
${ }^{c}$ Since $\exp \left(-E_{\text {bind }} / T\right)=\exp \left(\sum_{i} m_{u} / T\right) \cdot \exp \left(-\left(M_{h}-\right.\right.$ $\left.\left.\sum_{i}\left(m_{q_{i}}-m_{u}\right)\right) / T\right)$, the factor $\exp \left(\sum_{i} m_{u} / T\right)$ can be absorbed in $C$ and $C_{B}$. The error function of the fit is mainly
} 
Table 2: Results of the fit to LEP data and to data obtained at different center-of-mass energies.

\begin{tabular}{|l|l|l|}
\hline Parameters & $\sqrt{s}=91 \mathrm{GeV}$ & Simultaneous Fit \\
\hline \hline$\gamma_{s}$ & $0.29 \pm 0.03$ & $0.29 \pm 0.02$ \\
\hline$\Delta m(\mathrm{GeV})$ & $0.150 \pm 0.029$ & $0.161 \pm 0.024$ \\
\hline$T(\mathrm{GeV})$ & $0.289 \pm 0.020$ & $0.298 \pm 0.015$ \\
\hline$C$ & $0.209 \pm 0.041$ & $C_{91}=0.218 \pm 0.034{ }^{*}$ \\
\hline$C_{B}$ & $10.0 \pm 1.0$ & $11.0 \pm 0.9$ \\
\hline \hline$\chi^{2} /$ dof & $56.2 / 19$ & $155.8 / 57$ \\
\hline \multicolumn{2}{|c|}{$C_{30}=0.124 \pm 0.020}$, & $C_{10}=0.049 \pm 0.008$
\end{tabular}

The results of the fit to the LEP data for a typical s quark mass of $0.5 \mathrm{GeV}$ are listed in Table 2. The calculated total rate and primary fraction for different hadrons are listed in Table 1. We see a good agreement between the measured and calculated rates except for some decuplet baryons. As mentioned in Ref.0.6, experimental errors for the decuplet baryons are still large and there are discrepancies between experiments. The large $\chi^{2}$ value of the fit is mainly due to the decuplet baryons. If they are excluded in the fit, the $\chi^{2} / d o f$ is then equal to $22.6 / 15$ while the fit results remain essentially unchanged. However, the difference between data and our predictions for the decuplet baryons might suggest that baryon production is not described properly in our approach, since baryons are considerably more complifated objects than mesons. If diquark productiont is the main mechanism for baryon production, it is then more suitable to use the effective diquark masses than the quark masses in Eq.1. This will, however, increase considerably the number of free parameters.

We also perform a simultaneous fit to data at different center-of-mass energies with 7 free parameters: $\gamma_{s}, \Delta m, T, C_{B}, C_{91}, C_{30}$ and $C_{10}$, where $C_{91}, C_{30}$ and $C_{10}$ are the overall normalization factor at the center-of-mass energy 91, 29-35 and $10 \mathrm{GeV}$, respectively. The fit results are listed in Table 2 and shown in Fig. 2. The value of $\gamma_{s}$, $\Delta m, T$ and $C_{B}$ is very consistent with that obtained from the LEP data alone, showing that the value of these parameters is independent of the center-of-mass energy.

The $\gamma_{s}$ value determined from the fit is in an excellent agreement with the expectation. The value of $\Delta m=m_{s}-m_{u}$ required from the fit is consistent with that obtained from hadron masses 10 and from QCD calculations 12 . While the values of $\gamma_{s}, \Delta m$ and $T$ do not depend on the choice of the $m_{s}$ value, the values of $C$ and $C_{B}$ are

sensitive to the change in the mass difference $m_{s}-m_{u}$.

${ }^{d}$ The new result for $\Lambda(1520)$ is not included in the fit.

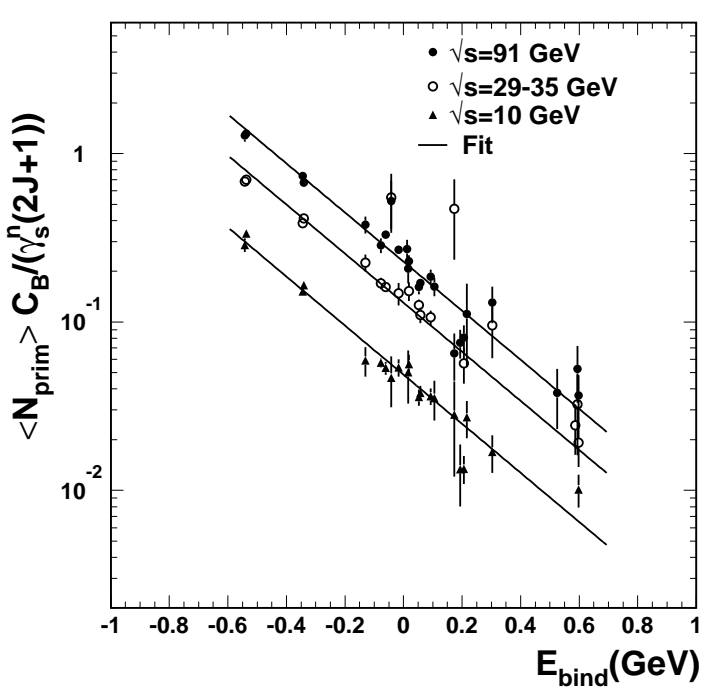

Figure 2: Production rates of light flavored hadrons originating from fragmentation at different center-of-mass energies, multiplied by the factor $C_{B} /\left(\gamma_{s}^{N_{s}}(2 J+1)\right)$, as a function of the binding energy of hadrons.

correlated with the $m_{s}$ value. However, the calculated baryon rates do not depend on the choice of the $m_{s}$ value since they are determined by both $m_{s}$ and $C_{B}$. From our analysis we find that baryons contribute $10.9 \%$ of the primary produced hadrons at LEP energy.

Fractions of primary hadrons determined from the fit are listed in Table 1. They are in general lower than those predicted by JETSET due to the inclusion of orbitally excited states up to a mass of $2.5 \mathrm{GeV}$ in our analysis. JETSET includes some of orbitally excited meson states (only those with $L=1$ ), but no orbitally excited baryon states. From our analysis we obtain the fraction of primary produced states with orbital excitation to be $27 \%$ for mesons and $39 \%$ for baryons at LEP energy.

Using the $T$ value obtained from the fit, the ratio $V /(V+P)$ can be calculated according to the mass difference of vector and pseudoscalar mesons. We obtain a value of $0.38,0.44,0.65$ and 0.72 for $\mathrm{u}(\mathrm{d})-$, s-, c- and b-mesons respectively, which are in good agreement with the measurements.

\section{Predictions of heavy flavor composition}

Using the fit results obtained with light flavored hadrons, the relative production rate of heavy flavored hadrons can be predicted by our method. The absolute value of the rates can be determined by normalizing the sum of the rates to the 
Table 3: Predictions of average production rates for heavy flavored hadrons, compared with LEP data.

\begin{tabular}{|c|c|c|}
\hline Hadrons & Prediction & Measurement \\
\hline $\mathrm{D}^{0}$ & 0.242 & $0.221 \pm 0.012$ \\
\hline $\mathrm{D}^{+}$ & 0.092 & $0.087 \pm 0.008$ \\
\hline $\mathrm{D}^{*+}$ & 0.114 & $0.088 \pm 0.006$ \\
\hline $\mathrm{D}_{s}^{+}$ & $0.054( \pm 0.005)^{a)}$ & $0.041 \pm 0.008$ \\
\hline$\Lambda_{c}^{+}$ & 0.026 & $0.037 \pm 0.009$ \\
\hline $\mathrm{B}^{0}$ & 0.091 & $0.097 \pm 0.026$ \\
\hline $\mathrm{D}^{*} /\left(\mathrm{D}^{*}+\mathrm{D}\right)^{b)}$ & 0.66 & $0.56 \pm 0.4$ \\
\hline $\mathrm{B}^{*} /\left(\mathrm{B}^{*}+\mathrm{B}\right)$ & 0.70 & $0.75 \pm 0.04$ \\
\hline \multicolumn{3}{|c|}{$<\mathrm{D}_{1}^{0}+\mathrm{D}_{2}^{* 0}>_{c} \operatorname{Br}\left(\mathrm{D}^{* * 0} \rightarrow \mathrm{D}^{*+} \pi^{-}\right) /<\mathrm{D}^{*+}>_{c}{ }^{b)}$} \\
\hline \multirow{4}{*}{$\begin{array}{l}\mathrm{B}_{u, d}^{* *} / \mathrm{B}_{u, d} \\
\left(\mathrm{~B}_{1}+\mathrm{B}_{2}^{*}\right)_{u, d} / \mathrm{B}_{u, d} \\
\left(\mathrm{~B}_{s 1}+\mathrm{B}_{s 2}^{*}\right) / \mathrm{B}_{u, d}^{* *} \\
\left(\mathrm{~B}_{s 1}+\mathrm{B}_{s 2}^{*}\right) / \mathrm{B}^{+}\end{array}$} & 0.39 & $(0.24 \pm 0.03) f^{c)}$ \\
\hline & 0.228 & $0.216 \pm 0.033$ \\
\hline & 0.110 & $0.142 \pm 0.055$ \\
\hline & 0.086 & $0.052 \pm 0.016$ \\
\hline $\mathrm{D}^{0} / \mathrm{c}$-jet & 0.593 & $0.570 \pm 0.046$ \\
\hline $\mathrm{D}^{+} / \mathrm{c}$-jet & 0.237 & $0.249 \pm 0.026$ \\
\hline $\mathrm{D}^{*+} / \mathrm{c}-$ jet & 0.272 & $0.241 \pm 0.015$ \\
\hline $\mathrm{D}_{s}^{+} / \mathrm{c}$-jet & $0.101( \pm 0.025)^{a)}$ & $0.128 \pm 0.027$ \\
\hline $\mathrm{B}_{s}^{0} / \mathrm{b}$-jet & $0.108( \pm 0.030)^{d)}$ & $0.122 \pm 0.031$ \\
\hline$\Lambda_{c}^{+} / \mathrm{c}$-jet & 0.069 & $0.076 \pm 0.044$ \\
\hline$\Lambda_{b} / \mathrm{b}$-jet & 0.073 & $0.076 \pm 0.019^{e)}$ \\
\hline$\left(\Sigma_{b}+\Sigma_{b}^{*}\right) /$ b-jet & $0.060^{f)}$ & $0.048 \pm 0.016$ \\
\hline $\mathrm{D}_{s 1} / \mathrm{c}-\mathrm{jet}$ & 0.012 & $0.016 \pm 0.006$ \\
\hline$\left(\mathrm{D}_{s 1}+\mathrm{D}_{s 2}^{*}\right) / \mathrm{c}$-jet & 0.028 & - \\
\hline$\left(\mathrm{B}_{s 1}+\mathrm{B}_{s 2}^{*}\right) / \mathrm{b}-$ jet & 0.035 & $0.037 \pm 0.012$ \\
\hline$\left(\mathrm{D}_{1}+\mathrm{D}_{2}^{*}\right)_{u, d} / \mathrm{c}$-jet & 0.170 & $0.173 \pm 0.053$ \\
\hline$\left(\mathrm{B}_{1}+\mathrm{B}_{2}^{*}\right)_{u, d} / \mathrm{b}$-jet & 0.188 & - \\
\hline $\mathrm{D}^{* *} / \mathrm{c}-\mathrm{jet}$ & 0.38 & - \\
\hline $\mathrm{B}^{* *} / \mathrm{b}-$ jet & 0.38 & - \\
\hline c-baryon/c-jet & 0.089 & - \\
\hline b-baryon/b-jet & 0.091 & $0.115 \pm 0.040$ \\
\hline \multicolumn{3}{|c|}{ a) for $\operatorname{Br}\left(\mathrm{D}_{s}^{* *} \rightarrow \mathrm{D}_{s} X\right)=0.5( \pm 0.5)$} \\
\hline \multicolumn{3}{|c|}{ b) excluding $\mathrm{D}, \mathrm{D}^{*}$ a $\mathrm{D}^{* *}$ from $\mathrm{B}$ decays } \\
\hline \multicolumn{3}{|c|}{ c) $f=1-2$ (see Ref. } \\
\hline \multicolumn{3}{|c|}{ d) for $\mathrm{Br}\left(\mathrm{B}_{s}^{* *} \rightarrow \mathrm{B}_{s} X\right)=0.5( \pm 0.5)$} \\
\hline \multicolumn{3}{|c|}{ e) for $\operatorname{Br}\left(\Lambda_{b}^{s} \rightarrow \Lambda_{c}^{+} l^{-} \bar{\nu} X\right)=0.1$} \\
\hline \multicolumn{3}{|c|}{${ }^{f)}=0.052$ if $m_{\Sigma_{b}, \Sigma_{b}^{*}}-m_{\Lambda_{b}}$ measured in Ref.14 are used } \\
\hline
\end{tabular}

$\Gamma_{c \bar{c}} / \Gamma_{h a d}$ or $\Gamma_{b \bar{b}} / \Gamma_{h a d}$, which can be calculated by the Standard Model. We use all heavy flavored hadrons in the JETSET 7.4 table, which includes orbitally excited mesons with $L=1$, such as $\mathrm{D}^{* *}$ and $\mathrm{B}^{* *}$, but no orbitally excited heary flavored baryons (for more discussions see Ref.9).

As an example we consider the heavy flavor composition at LEP energy. Our predictions to gether with the corresponding measurements 13 , are listed in Table 3. There is a good agreement between the predicted and measured values. The main uncertainty on the prediction is due to the limited knowledge on properties of orbitally excited heavy flavored hadrons, such as mass, decay modes, and branching ratios $\mathrm{g}$. Without taking into account the production of orbitally excited cand b-baryons, the fractions c-baryon/c-jet and bbaryon/b-jet are calculated to be around $9 \%$. The calculated values would be $30-40 \%$ higher 9 if orbitally excited c- and b-baryons could be included, which are however barely known.

\section{Conclusions}

We have shown that the production rates of light flavored mesons and baryons in $\mathrm{e}^{+} \mathrm{e}^{-}$annihilation, which span a range of four orders of magnitude, can be described by a simple approach based on the idea of string fragmentation. Data at different center-of-mass energies can be described simultaneously apart from a normalization factor, which reflects the rise of multiplicities with increasing energy. From the fit we determine the strangeness suppression factor to be $0.29 \pm 0.02$. Applying to the heavy flavor production, we find that our predictions are in good agreement with data. A comparison of our approach with other recently proposed approaches can be found in Ref. $\mathrm{E}$.

The proposed approach may provide insight into the hadron production mechanism. To further check the approach, precise data on the production of the decuplet baryons and orbitally excited hadrons are needed. In particular, with better understanding of the baryon production mechanism, the description of baryon production in our approach can be improved.

\section{References}

1. B. Andersson et al., Phys. Rep. 97 (1983) 31.

2. G. Marchesini and B.R. Webber, Nucl. Phys. B238 (1984) 1.

3. T. Sjöstrand, Comp. Phys. Comm. 82 (1994) 74; CERN-TH/93-7112.

4. G. Marchesini et al., Comp. Phys. Comm. 67 (1992) 465.

5. T. Sjöstrand, Preprint LU TP 95-19.

6. Y.J. Pei, L3 Internal Note 1912, a slightly modified version can be found in CERN Report CERN 96-01, Vol. 2, 109.

7. A. De Angelis, CERN-PPE/95-135.

8. OPAL Collab., G. Alexander et al., CERNPPE/96-099.

9. Y.J. Pei, CERN-PPE/96-37 (to appear in Z. Phys. C) and references therein.

10. G. Gasiorowicz and J.L. Rosner, Am. J. Phys. 49 (1981) 954.

11. Particle Data Group, Phys. Rev. D50 (1994).

12. H. Leutwyler, CERN-TH/96-25.

13. OPAL Collab., G. Alexander et al., Contributed paper Pa01-013 to ICHEP96;

ALEPH Collab., D. Buskulic et al., Phys. Lett. B357 (1995) 685; Contributed paper Pa01-075 to ICHEP96.

14. DELPHI Collab., P. Abreu et al., Contributed paper \#565 to EPS HEP95. 